\title{
FLEXIBLE ROBOTIC ELECTRONIC SKIN WITH HIGH SENSITIVITY SENSOR ARRAYS
}

\author{
Dr. T. Vijayakumar, \\ Professor, \\ Department of ECE, \\ GNIT, Hyderabad, India. \\ Email: vishal_16278@yahoo.co.in
}

\begin{abstract}
In this paper, we propose a flexible robotic electronic skin (e-skin) with high sensitivity sensor arrays. The sensor arrays are designed and fabricated on thin flexible silicone film. It uses piezo-resistive material Samarian Monosulphide and graphite rods for interconnection between the layers. Along with the pressure sensing capability of SmS, temperature and humidity sensors are also integrated in the silicone layers along with photovoltaic thin layer cells that provide energy independence to the module. The data procured from the e-skin is transferred to the analog to digital converter unit and further, to the PC through USB interface for analysis.
\end{abstract}

Keywords: E-skin, Samarian Monosulphide, Photovoltaic cell, ADC, pressure sensor

\section{INTRODUCTION}

A network of complex biological sensory receptors in the human skin can transmit the mechanical stimuli from the surrounding environment to the brain. Skin can sense pressure, temperature, humidity and other complex environmental stimuli [1]. Several researchers have been fascinated by this function and have tried to implement it technologically as artificial skin with an array of sensors since 1970s. This network of sensors can obtain mechanical stimuli from external sources and transform it to readable electrical signals. Electronic skin (E-skin) has evolved in such a way that it can replicate the functions of biological skin to a great extent. This has led to the creation of advanced humanoid robots, electronic surgical gloves, artificial prosthetics and wearable health monitoring devices and much more. This translational property of electronic skin works on the piezoelectric mechanism.

The major properties to be kept in mind while designing an electronic skin is the ability to handle multiple mechanical stimuli, stretchability and compatibility with implementation in large area. The conventional strain gauge sensors can sense a constricted strain range $(\varepsilon<5 \%)$ due to the inelastic nature of integral metal and semiconductor constituents [2]. Pressure sensors with 3D compliance are best suited components for the 
manufacture of e-skin. Several micro-fabrication techniques are used for developing flexible and stretchable electronic components.

Tactile and temperature sensing is a critical component for an e-skin structure. Along with this, implementation of self-healing property of human skin in e-skin is a great challenge. Self-healing materials such as metallosupramolecular polymer and repeatable thermoplastic elastomer are being fabricated based on its property to form dynamic reversible bonds [3]. Organic electronics has made their way into large area devices like e-skins and solar cells due to their light weight, low processing cost and intrinsic deformability. But they impose certain drawbacks in humid and oxygen-rich environments. Organic Field Effect Transistors (OFET) offers high tractability and low manufacturing cost, whereas, it offers lesser mobility and involves large operating voltage when compared to inorganic semiconductors. Recent progress in this field includes a combination of smart sensing along with wireless data transfer that is convenient for processing the obtained signals. Several researches are being done on fabricating extremely smart e-skins that respond to external stimuli with a minimal response time [1].

\section{RELATED WORK}

Various researchers from across the globe have conducted diverse research in the design, manufacture, and implementation and testing of e-skins. Lu, Nanshu et al. [4] proposed flexible e-skins that use Electro Active Polymer (EAP) that is used in soft actuation. EAPs are advantageous over the commonly used piezoelectric materials in terms of flexibility, response speed, hysteresis and strain levels. Huang et al. [5] uses the paper cutting art - kirigami to design stretchable transparent electrodes. This technology is used in the implementation of e-kin, bio inspired soft robotics and implantable biodegradable devices.

Zhang et al. [6] developed a stretchable dual-mode sensor array for e-skin that has cross-grid liquid metal layer of electrodes that are separated by micro-structured dielectric layers. These sensors can act as proximity sensors or pressure sensors and can be used for tactile sensing applications. Zhong et al. [7] proposed a nanofiber based wireless pressure sensor that acts as a signal transmitter along with Bluetooth technology. Fan et al. [8] used moulds that were made of lithographically patterned hard and soft substrates to create precise topographical microstructures of flexible patterned polymeric films and membranes.

Gong et al. [9] developed a simple and proficient, cost effective methodology to fabricate ultra-thin strain sensors using gold nano-wires with excellent elasticity and sensitivity. This fabrication was done on a prestretching latex rubber so as to further increase the stretchability of the sensor. The durability of the sensors were tested by observing the cracking of the film during strain and self -repairing capability on complete strain release.

ISSN: 2582-3825 (online) 


\section{PROPOSED WORK}

The proposed e-skin consists of layers of photo-resistive material, temperature and humidity sensors sandwiched in layers of flexible silicone film interconnected with graphene rods. The system has an independent power source. The data procurement unit is also modelled for acquisition of data from the e-skin.

\subsection{Sensor Design}

A thin membrane of flexible Silicone Film is used for the micro-fabrication of the sensor to measure the stress level of the external stimulus. A layer of Samarian Monosulphide (SmS) is used as a piezoresistive layer. Gold connecting layers are used to realise a conducting medium. With the application of external stimulus, the resistance varies. Based on this variation in the level of resistance, the type of stimulus is identified and suitable response is provided. Figure 1 shows the layers of fabrication of e-skin pressure sensor. CAD tools are used for the modelling of this sensor. Several layers of this sensor can be encompassed in a single flexible silicon layer as a sandwich with adhesive material in between the layers. An array of these sensors can be used as the base for eskin.

$\mathrm{SmS}$ is a fascinating compound that exhibits a transition from semiconductor to metallic state on application of pressure [10]. This switching property makes it an ideal candidate in the use of e-skin sensors. The ratio between $\mathrm{SmS}$ and gold is chosen in such a way that it provides the essential viscidness, reduced surface tension and even coat. Thin film deposition techniques like Electro-deposition and Molecular Beam Epitaxy (MBE) are used in the manufacture of the sensor component.

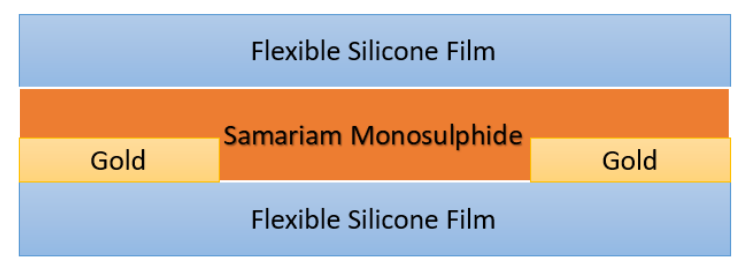

Figure 1 Layers of e-skin pressure sensor 
Gold offers uniquely high flexibility as well as conductivity and can be made as thin as $2 \mathrm{~nm}$ for fabrication purpose. Dropcasting technology can be used while using gold in the fabrication of the e-skin pressure sensors. This sensor can also be incorporated with existing fabric clothing. In order to manufacture a high resolution and highly elastic sensor array, each sensor component should be scale down to its smallest possible magnitudes.

\subsection{Temperature and Humidity Sensors}

Several elements such as Carbon Nano Tubes (CNT), Graphene and polymers are considered for the fabrication of temperature and humidity sensors [11]. Fabrication of elastic temperature sensor that offers steady performance on application of strain is challenging as temperature tends to change due to stretching. We use intrinsically transparent and stretchable components like reduced graphene oxide nanosheets in elastometric polyurethane matrix for fabricating the temperature sensor that can be easily integrated with the strain sensor [12] Along with this, we use the WS2 humidity sensor [13] due to its flexibility and skin-activity compatibility.

\subsection{Graphene Interconnects}

Printable and stretchable graphene Nano-composite materials are used for interconnection between the layers and components of e-skin. Dang et al. [14] performed a comparative study on the elastic property of various materials such as metals, polymers and carbon and found carbon to be the most optimal material of all. They offer good elasticity and electrical conductivity. Normal placing techniques would cause the carbon fillers to roll and interweave due to the strong Van der Wall forces and cause scattered conductivity. Hence lithographic technologies like micro-contact printing, inkjet printing or spray coating will be suitable for laying the Graphene interconnects. Graphene is an extensively used carbon filler for flexible electrodes. This is because they exhibit excellent conductivity with low filtration ratio due to its large surface area.

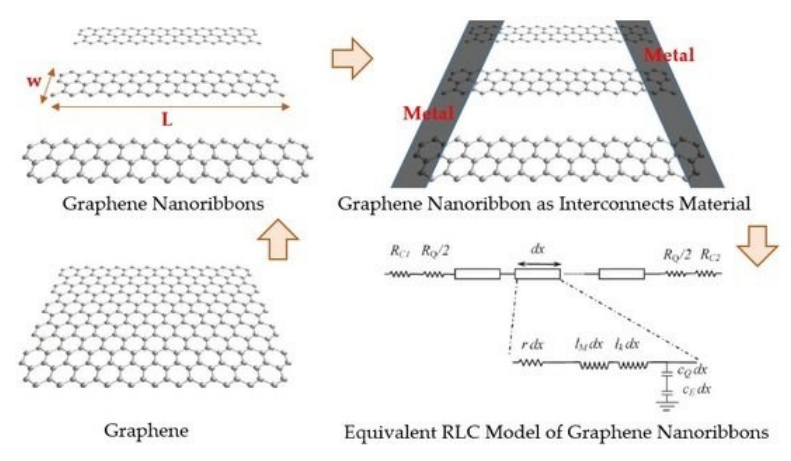

ISSN: 2582-3825 (online) 


\subsection{Power Source}

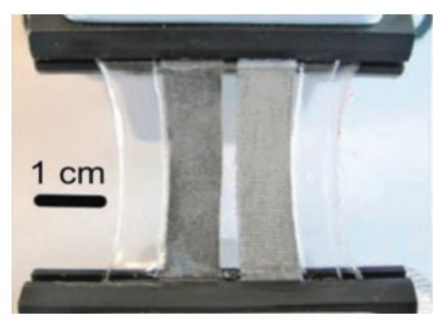

(a)

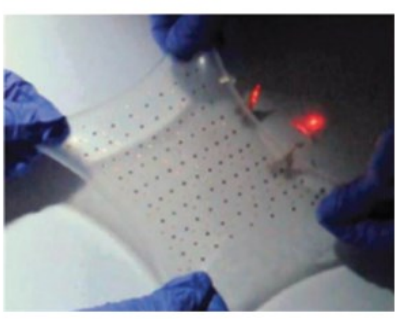

(b)

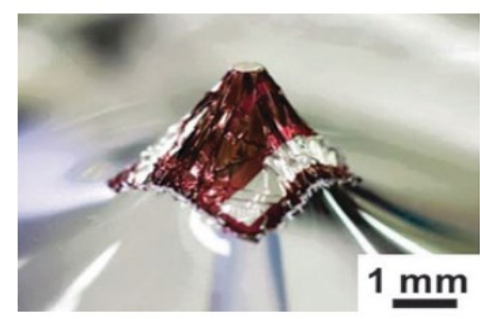

(c)

Figure 4 Stretchable Power Sources

Most suited power supply sources for e-skin includes wireless transmission, piezoelectric supply and photovoltaic sources. Figure 4 represents (a) The early effort of stretchable battery with electrochemically active materials embedded in compliant conductive fabrics [15] (b) Array of lithium-ion battery microcells interconnected by "self-similar" serpentine electrodes with stretchability up to 300\% [16] (c) stretchable solar cells based on wrinkled organic photovoltaic thin films on elastomer substrates [17].

Photovoltaic cell uses light energy for generation of power and can be manufactured by depositing photovoltaic material on substrates or other materials. It produces power at a very low cost and has a wide range of applications. We use the photovoltaic thin film power source in order to provide energy independence to the eskin model.

\subsection{Data Procurement Unit}




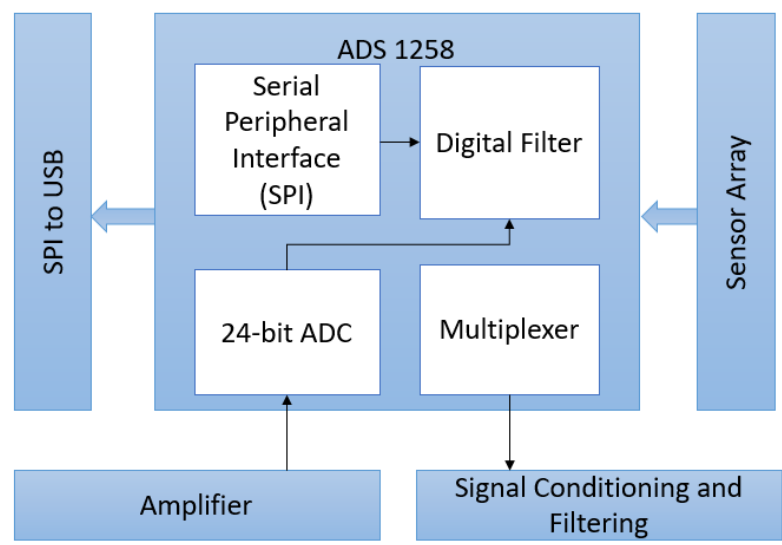

Figure 5 Data Procurement Unit Block Diagram

The data procurement unit is connected to the sensor array to calculate its response to the external stimuli. It contains signal conditioning, amplifying and regulatory circuit. It is represented as a block diagram in Figure 5. We use a 16 channel, 24-bit ADS1258 Analog to Digital Converter IC from Texas Instruments. It also consists of an inbuilt Serial Peripheral Interface (SPI), a digital filter and a multiplexer unit. The output of this system is fed to the amplifier and the amplified signal are fed back to the ADC. This amplified data is transferred to the computer through the USB port for further processing.

\section{RESULT}

The synthetic E-skin is designed and modelled using 3D CAD and fabricated on a flexible silicone film substrate with SmS and gold and sandwiched with adhesive material in between the layers. Further, photovoltaic cell, humidity and temperature sensors are also placed between the layers. Care is taken in such a way that the final e-skin material is maintained at the lowest possible thickness. Further, a data acquisition system is designed and the obtained output data from the e-skin is fed to the system through USB for further processing. The e-skin responds to the eternal stimuli at a delay of $75 \mathrm{~ms}$.

\section{CONCLUSION}

A synthetic e-skin with high-tenacity pressure sensing is fabricated. It is made up of a silicone and $\mathrm{SmS}$ based see-through solid sensitive layer and photovoltaic cell that provides energy independence. Humidity, temperature and pressure sensors were integrated into the layers through lamination. Graphene interconnects were used to connect between the layers. It can identify the variations in stimuli like wind, breath and touch. 
Journal of Electronics and Informatics (2019)

Vol.01/ No. 01

Pages: 43-51

http://www.irojournals.com/iroei/

DOI: https://doi.org/10.36548/jei.2019.1.005

Future work involves addition of more sensors and reducing the response time. Further, including features beyond human skin are also proposed to be implemented in e-skin such as stretchable electroluminescent polymers [18] and optical light emitting displays that can illuminate e-skin as well as display content on the skin.

\section{References}

[1] Wang, Xiandi, Lin Dong, Hanlu Zhang, Ruomeng Yu, Caofeng Pan, and Zhong Lin Wang. "Recent progress in electronic skin." Advanced Science 2, no. 10 (2015): 1500169.

[2] Barlian, A. Alvin, Woo-Tae Park, Joseph R. Mallon, Ali J. Rastegar, and Beth L. Pruitt. "Semiconductor piezoresistance for microsystems." Proceedings of the IEEE 97, no. 3 (2009): 513-552.

[3] White, Scott R., Nancy R. Sottos, Philippe H. Geubelle, Jeffrey S. Moore, M_R Kessler, S. R. Sriram, E. N. Brown, and S. Viswanathan. "Autonomic healing of polymer composites." Nature 409, no. 6822 (2001): 794

[4] Lu, Nanshu, and Dae-Hyeong Kim. "Flexible and stretchable electronics paving the way for soft robotics." Soft Robotics 1, no. 1 (2014): 53-62.

[5] Huang, Siya, Yuan Liu, Yue Zhao, Zhifeng Ren, and Chuan Fei Guo. "Flexible electronics: Stretchable electrodes and their future." Advanced Functional Materials 29, no. 6 (2019): 1805924.

[6] Zhang, Cheng, Shaoyu Liu, Xin Huang, Wei Guo, Yangyang Li, and Hao Wu. "A stretchable dual-mode sensor array for multifunctional robotic electronic skin." Nano Energy 62 (2019): 164-170.

[7] Zhong, Weibin, Qiongzhen Liu, Yongzhi Wu, Yuedan Wang, Xing Qing, Mufang Li, Ke Liu, Wenwen Wang, and Dong Wang. "A nanofiber based artificial electronic skin with high pressure sensitivity and 3D conformability." Nanoscale 8, no. 24 (2016): 12105-12112.

[8] Fan, Z., Razavi, H., Do, J.W., Moriwaki, A., Ergen, O., Chueh, Y.L., Leu, P.W., Ho, J.C., Takahashi, T., Reichertz, L.A. and Neale, S., 2009. Three-dimensional nanopillar-array photovoltaics on low-cost and flexible substrates. Nature materials, 8(8), p.648. 
Journal of Electronics and Informatics (2019)

Vol.01/ No. 01

Pages: 43-51

http://www.irojournals.com/iroei/

DOI: https://doi.org/10.36548/jei.2019.1.005

[9] Gong, Shu, Daniel TH Lai, Bin Su, Kae Jye Si, Zheng Ma, Lim Wei Yap, Pengzhen Guo, and Wenlong Cheng. "Highly Stretchy Black Gold E- Skin Nanopatches as Highly Sensitive Wearable Biomedical Sensors." Advanced Electronic Materials 1, no. 4 (2015): 1400063.

[10] Jarrige, Ignace, Hitoshi Yamaoka, J-P. Rueff, J-F. Lin, Munetaka Taguchi, Nozomu Hiraoka, Hirofumi Ishii et al. "Unified understanding of the valence transition in the rare-earth monochalcogenides under pressure." Physical Review B 87, no. 11 (2013): 115107.

[11] Hong, Soo Yeong, Yong Hui Lee, Heun Park, Sang Woo Jin, Yu Ra Jeong, Junyeong Yun, Ilhwan You, Goangseup Zi, and Jeong Sook Ha. "Stretchable active matrix temperature sensor array of polyaniline nanofibers for electronic skin." Advanced Materials 28, no. 5 (2016): 930-935.

[12] Trung, Tran Quang, Subramaniyan Ramasundaram, Byeong- Ung Hwang, and Nae- Eung Lee. "An allelastomeric transparent and stretchable temperature sensor for body- attachable wearable electronics." Advanced materials 28, no. 3 (2016): 502-509.

[13] Guo, Huayang, Changyong Lan, Zhifei Zhou, Peihua Sun, Dapeng Wei, and Chun Li. "Transparent, flexible, and stretchable WS 2 based humidity sensors for electronic skin." Nanoscale 9, no. 19 (2017): 6246-6253.

[14] Dang, Wenting, Vincenzo Vinciguerra, Leandro Lorenzelli, and Ravinder Dahiya. "Printable stretchable interconnects." Flexible and Printed Electronics 2, no. 1 (2017): 013003.

[15] Gaikwad AM, Zamarayeva AM, Rousseau J, Chu HW, Derin I, Steingart DA. Highly stretchable alkaline batteries based on an embedded conductive fabric. Adv Mater 2012; 24:5071-5076.

[16] Xu S, Zhang YH, Cho J, Lee J, Huang X, Jia L, et al. Stretchable batteries with self-similar serpentine interconnects and integrated wireless recharging systems. Nat Commun 2013; 4:1543.

[17] Kaltenbrunner M, White MS, Glowacki ED, Sekitani T, Someya T, Sariciftci NS, Bauer S. Ultrathin and lightweight organic solar cells with high flexibility. Nat Commun 2012; 3:770.

[18] Liang, Jiajie, Lu Li, Xiaofan Niu, Zhibin Yu, and Qibing Pei. "Elastomeric polymer light-emitting devices and displays." Nature Photonics 7, no. 10 (2013): 817. 
Journal of Electronics and Informatics (2019)

Vol.01/ No. 01

Pages: 43-51

http://www.irojournals.com/iroei/

DOI: https://doi.org/10.36548/jei.2019.1.005

[19] Saadatzi, Mohammad Nasser, Joshua R. Baptist, Zhong Yang, and Dan O. Popa. "Modeling and Fabrication of Scalable Tactile Sensor Arrays for Flexible Robot Skins." IEEE Sensors Journal (2019).

[20] Ma, Mingyuan, Zheng Zhang, Qingliang Liao, Fang Yi, Linhong Han, Guangjie Zhang, Shuo Liu, Xinqin Liao, and Yue Zhang. "Self-powered artificial electronic skin for high-resolution pressure sensing." Nano Energy 32 (2017): 389-396.

[21] Ge, Jin, Li Sun, Fu- Rui Zhang, Ye Zhang, Lu- An Shi, Hao- Yu Zhao, Hong- Wu Zhu, Hai- Long Jiang, and Shu- Hong Yu. "A stretchable electronic fabric artificial skin with pressure- , lateral strain- , and flexionsensitive properties." Advanced Materials 28, no. 4 (2016): 722-728.

[22] Ho, Dong Hae, Qijun Sun, So Young Kim, Joong Tark Han, Do Hwan Kim, and Jeong Ho Cho. "Stretchable and multimodal all graphene electronic skin." Advanced Materials 28, no. 13 (2016): 2601-2608. 